\title{
Symmetrical Facial Giant Plaque-Type Juvenile Xanthogranuloma: Case Report and Review of the Literature
}

\author{
Kaspar Itin Peter Häusermann Peter Itin Nicole Fosse \\ Department of Dermatology, University Hospital Basel, Basel, Switzerland
}

\section{Keywords}

Juvenile xanthogranuloma $\cdot$ Symmetrical facial plaque-type JXG $\cdot$ Non-Langerhans

histiocytosis · Touton giant cells · CD68

\begin{abstract}
Juvenile xanthogranuloma (JXG) is the most common type of non-Langerhans cell histiocytosis. JXG is a rare benign tumor, which may be present at birth or develop later. The classical form of JXG is characterized by a red-yellowish benign papule or nodule with predilection sites on the head, neck, and trunk, although lesions can appear on extremities or extracutaneous sites. In most cases there is only one lesion, whereas numerous papules or nodules may occur. Special forms of JXG such as mixed, giant, subcutaneous, eruptive, clustered, and plaque-like have been reported and associations between JXG and systemic diseases have been made. Diagnosis mainly relies on the clinical appearance, and histology usually can confirm the disease. Here we present a very rare case of symmetrical giant facial plaque-type juvenile xanthogranuloma (SGFP-JXG) and compare it with classical JXG, variations of JXG, and discuss the differential diagnosis. A 4-year-old Caucasian female presented with plaque-like lesions composed of yellowish confluent papules on both the cheeks. The histological evaluation revealed a histiocytic lesion with a formation of Touton giant cells and immunohistochemistry results confirmed the diagnosis of the SGFP-JXG. In comparison to classical JXG, the onset of SGFP-JXG sometimes occurs later and the spontaneous resolution period may be prolonged. No associated diseases and no systemic involvements were observed. Histopathology is required to differentiate this form of JXG from other histiocytosis. To the best of our knowledge, only four cases of SGFP-JXG have been reported in the literature so far.
\end{abstract}




\section{Introduction}

Juvenile xanthogranuloma (JXG) is the most common type of non-LCH and is considered a rare disease that may be present at birth or acquired later [1]. Most cases of JXG appear during the first year of life [2]. The classical form of JXG is characterized by a red-yellowish benign papule $(<0.5 \mathrm{~cm})$ or nodule $(<2 \mathrm{~cm})$ that mainly involves the head and neck $(42 \%)$ as well as trunk $(20-40 \%)$, although the lesion can occur at any sites of the body, including extracutaneous areas $[2,3]$. Special forms of JXG such as mixed, giant, subcutaneous, eruptive, clustered, and plaque-like have been reported [1]. Lesions found with a diameter $>2 \mathrm{~cm}$ are termed giant or macronodular. Symmetrical giant facial plaque-type juvenile xanthogranuloma (SGFP-JXG) is an uncommon type of xanthogranuloma, which was first described by Gunson and Birchall [4] in 2008.

Associations between JXG and neurofibromatosis, Niemann-Pick disease, urticaria pigmentosa, and myeloid leukemia have been documented [2]. Diagnosis mainly relies on clinical appearance, but in some cases a biopsy is required for the confirmation. Collections of histiocytes, foam cells, and Touton giant cells can be identified histologically in JXG [5]. Cutaneous lesions usually are self-limiting with spontaneous resolution, though some extracutaneous can be associated with morbidity $[2,6]$.

\section{Case Presentation}

Initially a 2-year-old Caucasian female presented herself with newly emerged yellowish to lightly brown papules on both the cheeks. The differential diagnoses suggested by the dermatologist included xanthoma-like dermatosis induced by abnormalities of lipid metabolism, xanthogranuloma through histiocytosis, or a granulomatous disorder. Therapy with a topical glucocorticosteroid cream did not bring any improvement. Over time, the lesions became larger and at the age of 3-and-a-half years, the girl was referred to a pediatric clinic. At that time, the lesions were described as symmetrical, multiple, confluent lightly brownish-orange soft papules to plaques which spread over an area of $4 \times 5 \mathrm{~cm}$ on both the cheeks. Few and small lightly brownish-orange papules were localized in the periorbital region. The rest of integument was normal. The suspected plane xanthoma was treated with trichloroacetic acid 30\% and silversulfadiazin wound cream. After two follow-up consultations, there were no signs of amelioration. 5 months later, the family showed up at the University Hospital of Basel for a further opinion. Clinical investigation showed a $7 \times 5 \mathrm{~cm}$ plaque on the right cheek and a second $4 \times 3 \mathrm{~cm}$ lesion on the left cheek (shown in Fig. 1 , $2 a)$ composed of yellowish confluent papules with a central and edgy macular proportion. The Darier sign was negative and there was no lymphadenopathy in the locoregional area. Except for these asymptomatic skin lesions and a treated strabismus divergens, the child was otherwise healthy. Next, a biopsy was done and histological evaluation revealed a histiocytic lesion with formation of Touton giant cells (shown in Fig. 3a) and without any sign of Langerhans-cell histiocytosis, mastocytosis, and melanocytic lesions. Immunohistochemistry yielded histiocytic cells immunoreactive for mainly CD68 (shown in Fig. 3b), some for Factor XIIIa but not for CD1a. HMB45 and S-100 were negative too. According to the histological results, diagnosis of SGFP-JXG was made. 4 months later, marked spontaneous amelioration was noted (shown in Fig. 2b).

\section{Discussion}

JXG belongs to proliferative dendric cell-related disorders, and it is considered a form of non-Langerhans histiocytosis. It is the most common member of this disease family [1]. In JXG, a male predominance (male to female ratio, 1.1-7:1) has been registered [3]. Clinically,

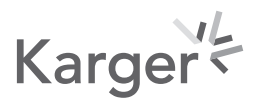


Fig. 1. Bilateral lesions: 2-and-a-half years after the first appearance.
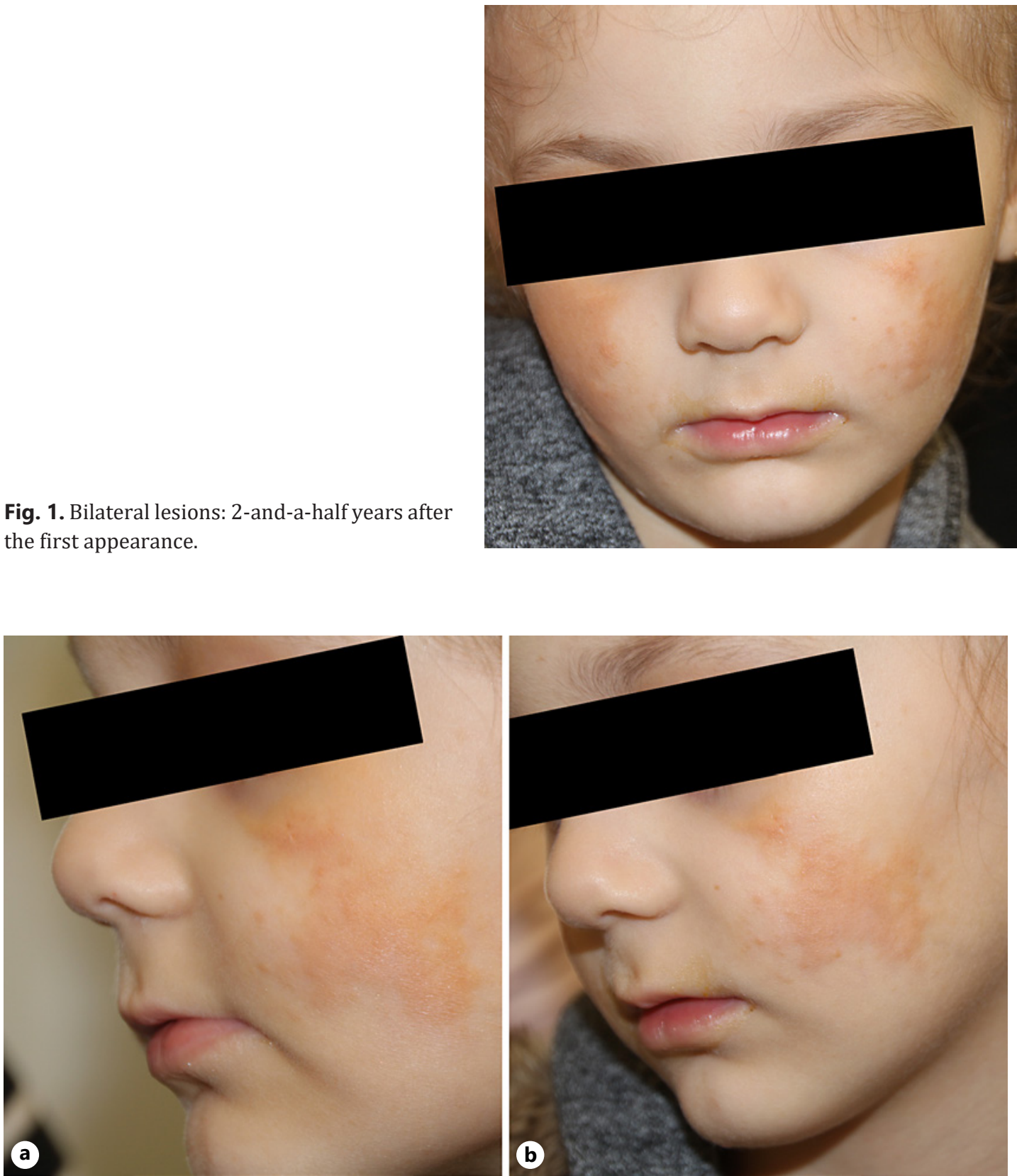

Fig. 2. Clinical improvement on the left cheek between the first consultation (a) and the follow-up 4 months later at the University Hospital of Basel (b).

it usually manifests itself as a slowly growing, asymptomatic, reddish-brown papule or nodule, which often evolves into a yellowish-brown papule, nodule, or plaque [2,3]. The cutaneous lesion usually disappears spontaneously within 3-5 years [5, 6]. Atypical clinical forms including keratotic, lichenoid, pedunculated, subcutaneous, clustered, intramuscular, plaquetype, and giant lesions seem to be very rare [7-9]. To the best of our knowledge, only eight plaque-type JXGs have been reported in the literature [4, 8-14], of which four cases have been reported as SGFP-JXG [4, 12-14]. The present case is the fifth SGFP-JXG. The pathogenesis remains unclear, although it is suggested that a trauma or infection triggers might induce a reactive proliferative process of histiocytes [15]. 

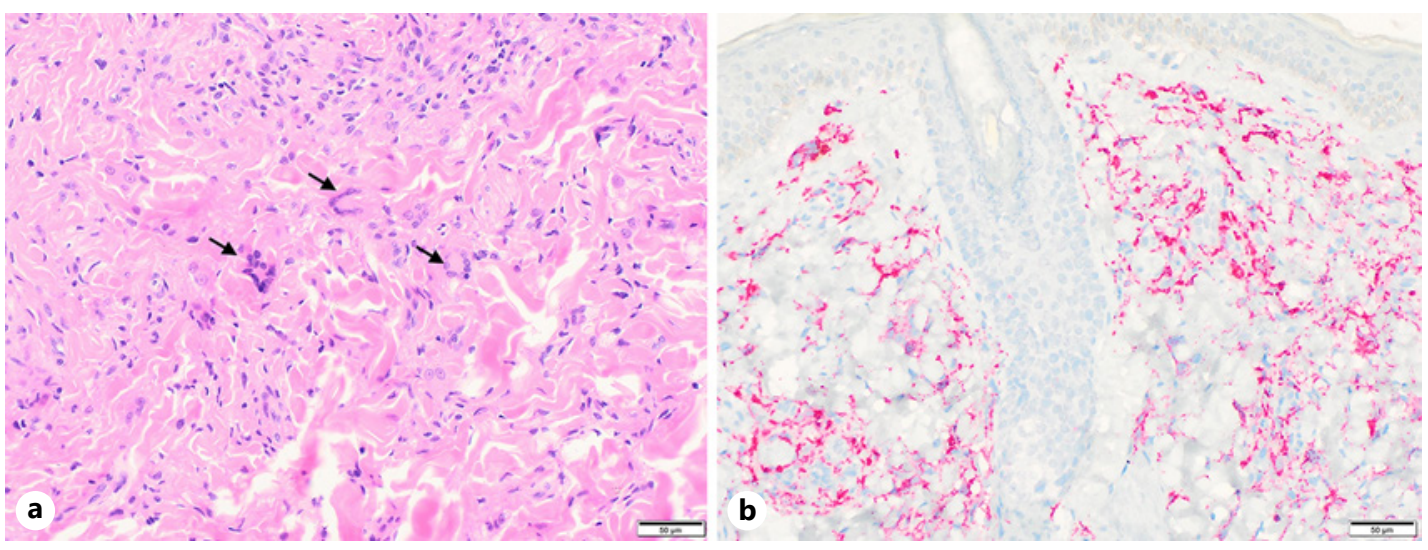

Fig. 3. a Hematoxylin-eosin staining of the yellowish plaque on the right cheek. Numerous Touton giant cells are seen as indicated by arrows. b Immunohistochemical staining shows that histiocytes have a positive reaction to $\mathrm{CD} 68$.

\section{Natural History}

In classical JXG, about $10 \%$ of the cases are present at birth, infants and small children are most often affected, and 85\% of the cutaneous lesions occur before the age of 1 year [16]. Compared to the classical JXG, plaque-type JXG sometimes appears beyond the first year of life [2]. In SGFP-JXG, we describe the second case with the first signs of the rash around the second year of life [14]. No correlation between ethnicity and the incidence of SGFP-JXG has been observed.

\section{Localization}

Lesions are solitary in two-thirds of the cases with systemic involvement occurring in $<20 \%[1,6]$. Ocular involvement is most commonly extracutaneous manifestation. The incidence of ocular complications in patients with cutaneous JXG is reported in the literature from 0.24 to $10 \%$ [17-19]. Ocular features include iris lesions, spontaneous hyphema, uveitis, heterochromia, and rarely posterior segment involvement. Secondary glaucoma has been recognized as a common complication and cause of severe visual loss in patients with JXG [17]. In order to minimize the complications of secondary glaucoma in ocular JXG, early diagnosis and intervention is required. The utility of routine screening is not well established. Based on the risk factors for ocular complications in patients with JXG as described in the literature, we recommend an ophthalmological screening for those patients with multiple skin lesions who are $<2$ years of age [18] or with periocular skin lesions [20]. Our patient's periorbital region was affected by the lesions, so an ophthalmological examination was made which revealed no abnormalities. Other extracutaneous locations, such as the lungs, liver, brain, spleen, bones, heart, adrenal glands, and mucus glands have also been reported in single cases $[1,7,15]$. Since most of these extracutaneous lesions in JXG heal spontaneously there is not any recommendation for a systemic screening in the literature, and it is reasonable to investigate for systemic disease only in the presence of symptoms [21,22]. The plaque-type JXG occurs most commonly on the upper trunk, arms, face, and head $[8,9]$. In SGFP-JXG, extrafacial cutaneous sites with JXG were described, in 1 case on the elbow [12], and in other case body regions, such as abdomen, back, and upper extremities were affected [14]. Furthermore, none of the SGFP-JXG cases observed internal organ involvements [4, 12-14].

\section{Karger'}




\section{Morphology and Clinical Manifestation}

Our patient presents with flat confluent giant plaques on both the cheeks, containing some solitary papules and nodules on the edges of the maculopapular plaque-type lesions. At the center of the plaque, we observed a more macular part. Different to the case reports of Gunson and Birchall [4], Sugiura et al. [12], and Sharquie et al. [14], the upper eyelids are spared, while the lower eyelids are slightly affected by macular changes. The nature of our patients lesions seem to differ to the case of Gunson and Birchall [4] describing a SGFP-JXG with a homogenous peau d'orange appearance on the surface. Our patient's lesions are smaller in size and the multiple flat plaques are composed of macular, papular, and nodular qualities, which form an inhomogeneous surface. Similar to the other cases, spared areas are well-demarcated [4, 12-14].

\section{Dynamic}

A JXG often evolves into a yellow-brown papule, plaque, or nodule, followed by a spontaneous resolution within 1-5 years, which may leave an atrophic scar, anetoderma, or hyperpigmentation [23]. The disease rarely persists beyond the late childhood [8]. Our patient had no spontaneous resolution 2 years after the disease started, although we observed a slight regression of the lesions over time. The four cases in the literature describing SGFP-JXG showed no regression over time for the patients aged 2-and-a-half [14], 3 [13], 7 [4], and 10 [12] years. Plaque and cluster types of JXG at extrafacial sites usually regress within 1 year with scarring and pigmentation despite the atypically large lesions [24,25]. We conclude that regression time in SGFP-JXG might be longer than in classical JXG and extrafacial plaque-type JXG.

\section{Therapy}

Due to the excellent prognosis of cutaneous lesions without visceral involvement a waitand-see strategy is recommended [7, 15]. Also extracutaneous JXG commonly regresses over time; treatment is only suggested in case of abnormal organ function [15]. Surgical resections for accessible symptomatic lesions have been performed and are generally curative, despite the fact that recurrence has been documented [23]. In facial JXG, the therapeutic options are limited, given the large and cosmetically sensitive area involved. In unresectable symptomatic visceral lesions, high dose steroids, LCH-based chemotherapy with vinca alkaloids, cyclosporine, and radiotherapy or a combination of the two have been used successfully $[6,7,15]$. Lee and Oh [13] described the first case report of a persistent SGFP-JXG on which a single fractional ablative laser therapy was performed with a significant reduction of the lesion.

\section{Associated Diseases}

JXG has been associated with neurofibromatosis (NF), Niemann-Pick disease, urticaria pigmentosa, and myeloid leukemia [2]. In 1937, Lamb and Lain [26] showed an existing association between JXG and NF1. Approximately half of NF1 cases occur sporadically, whereas JXG may be a cutaneous manifestation of NF1. A diagnosis of NF1 in children is established by the presence of at least 2 of 7 clinical criteria established by the National Institutes of Health, one criterion being the presence of 6 or more café au lait macules larger than $0.5 \mathrm{~cm}$ [27]. According to Caputo [28], CALM of NF may be noted in about $20 \%$ of the patients with JXG. Additionally Ferrari et al. [29] found a high frequency of JXG and naevus anaemicus in patients with NF1. Consequently, we looked for CALMs and naevus anaemicus in our patient, but no such findings were observed. Our patient did not fulfill any National Institutes of Health criteria. Cases with chronic urticaria and positive Darier signs have been reported and suggest a novel understanding of the interaction between dermal mast cells and dendrocytes [30]. Examination of the patient revealed a negative Darier sign and no findings that would imply LCH and Niemann-Pick disease.

\section{Karger'}




\section{Differential Diagnosis}

The diagnosis of JXG is mainly based on characteristic clinical features, although unusual presentations of JXG can pose significant diagnostic difficulties. The majority of differential diagnosis for SGFP-JXG refers to the other histiocytosis. Like benign cerebral histiocytosis, JXG is most common in children, but is usually easily differentiated by histopathology because only JXG has Touton giant cells [7]. The difficult part of diagnosing SGFP-JXG was differentiating it from diffuse normolipaemic planar xanthoma (DNPX) and xanthoma disseminatum, as these are often histologically indistinguishable from JXG. Both are expressions of a broad spectrum of histiocytic proliferative disorders. Xanthoma disseminatum shows the following triad: (1) papular or patchy xanthomatous lesions localized on flexures or eyelids, (2) diabetes insipidus in $40 \%$ of the cases due to hypothalamic/hypophyseal compromise, and (3) xanthomas on mucous membranes and in the upper respiratory tract. It mainly affects young male adults, who are normolipemic [31]. Also the diagnosis of DNPX is based on 3 major features: (1) diffuse plane xanthomas of the head, neck, trunk, and extremities, (2) palpebral xanthelasma, (3) normal plasma lipid levels [32]. DNPX typically shows characteristic xanthelasma appearing first in most cases, and tends to occur more frequently in adults [33]. Considering the typical clinical presentation of our case without preceding appearance of xanthelasma, the patient's age, and its greater degree of similarity with the previously reported cases of SGFP-JXG, favors the current diagnosis.

\section{Conclusion}

We report a very rarely described SGFP-JXG presenting with a typical clinical picture. The present patient is considered the fifth case to be reported. No spontaneous resolution has been observed in SGFP-JXG so far, but our case is the first one to show signs of regression. Plaque-type JXG tends to occur in older children than classical form of JXG. Due to the benign course, no treatment is mandatory. Neither systemic involvement nor associated diseases were detected in our case. Histopathology may be required to differentiate other forms of histiocytic disorders.

\section{Statement of Ethics}

The authors state that the patient's parents gave written informed consent for the case to be published (including publication of images). This research complies with all Ethical Guidelines for human studies in accordance with the World Medical Association Declaration of Helsinki.

\section{Conflict of Interest Statement}

The authors have no conflicts of interest to declare.

\section{Funding Sources}

There was no funding for this case report.

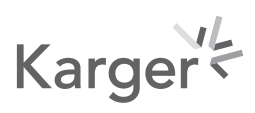




\section{Author Contributions}

K.I.: conception and design, analysis and interpretation of the data, and wrote the initial manuscript draw. P.I.: conception and design, acquisition of the data, and critically revising the work. P.H.: acquisition of the data and critically revising the work. N.F.: acquisition of data and critically revising the work. All authors have given the final approval of the version to be published and agree to be accountable for all aspects of the work in ensuring that questions related to the accuracy or integrity of any part of the work are appropriately investigated and resolved.

\section{References}

1 Hernandez-Martin A, Baselga E, Drolet BA, Esterly NB. Juvenile xanthogranuloma. J Am Acad Dermatol. 1997 Mar;36(3 Pt 1):355-9.

2 Dehner LP. Juvenile xanthogranulomas in the first two decades of life: a clinicopathologic study of 174 cases with cutaneous and extracutaneous manifestations. Am J Surg Pathol. 2003 May;27(5):579-93.

3 Janssen D, Harms D. Juvenile xanthogranuloma in childhood and adolescence: a clinicopathologic study of 129 patients from the kiel pediatric tumor registry. Am J Surg Pathol. 2005 Jan;29(1):21-8.

4 Gunson TH, Birchall NM. Symmetrical giant facial plaque-type juvenile xanthogranuloma. J Am Acad Dermatol. 2008 Aug;59(2 Suppl 1):S56-7.

5 Sangüeza OP, Salmon JK, White CR, Beckstead JH. Juvenile xanthogranuloma: a clinical, histopathologic and immunohistochemical study. J Cutan Pathol. 1995 Aug;22(4):327-35.

6 Freyer DR, Kennedy R, Bostrom BC, Kohut G, Dehner LP. Juvenile xanthogranuloma: forms of systemic disease and their clinical implications. J Pediatr. 1996 Aug;129(2):227-37.

7 Goodman W, Barrett T. Non-Langerhans cells histiocytoses. In: Bolognia J, Jorizzo J, Rapini R, editors. Bolognia dermatology. 2nd ed. Philadalphia: Mosby Elsevier; 2008. p. 1395-410.

8 Chang MW. Update on juvenile xanthogranuloma: unusual cutaneous and systemic variants. Semin Cutan Med Surg. 1999 Sep;18(3):195-205.

9 Caputo R, Cambiaghi S, Brusasco A, Gelmetti C. Uncommon clinical presentations of juvenile xanthogranuloma. Dermatology. 1998;197(1):45-7.

10 Lazova R, Shapiro PE. Juvenile xanthogranuloma versus langerhans cell histiocytosis (histiocytosis X). Semin Cutan Med Surg. 1999 Mar;18(1):71-7.

11 de Oliveira Rocha B, de Sousa Medeiros Torres I, de Almeida Rêgo VRP, Fernandes JD. Erythematous, yellowish plaque on the face of a child. Int J Dermatol. 2013;52(3):295-6.

12 Sugiura K, Hasegawa Y, Shimoyama Y, Hashizume H, Akiyama M. Symmetrical giant facial plaque-type juvenile xanthogranuloma persisting beyond 10 years of age. Acta Derm Venereol. 2014;94(4):465-6.

13 Lee YI, Oh S. Symmetrical giant facial plaque-type juvenile xanthogranuloma: a case report with a successful response to fractional CO2 laser treatment. Ann Dermatol. 2019 Jan;31:209.

14 Sharquie KE, Al-Jaralla FA, Al-Saadawi AR. Symmetrical giant facial plaque-type juvenile xanthogranuloma; a new case report. Am J Dermatol Venereol. 2019;8(2):33-5.

15 Chu AC. Histiozytoses. In: Burns T, Rook GA, Cox N, Griffiths C, editors. Rook's textbook of dermatology: in four volumes. 8th ed. Oxford: Wiley-Blackwell; 2010. p. 55.15-55.17.

16 Curtis T, Wheeler D, Cibis G, Law S, Rowsey J, Brown L, et al. Juvenile xanthogranuloma: background, pathophysiology, epidemiology [Internet]. 2019 Nov [cited 2020 Dec 29]. Available from: https://emedicine. medscape.com/article/1209681-overview.

17 Zimmerman LE. Ocular lesions of juvenile xanthogranuloma. Nevoxanthoedothelioma. Am J Ophthalmol. 1965 Dec;60(6):1011-35.

18 Chang MW, Frieden IJ, Good W. The risk intraocular juvenile xanthogranuloma: survey of current practices and assessment of risk. J Am Acad Dermatol. 1996 Mar;34(3):445-9.

19 Samuelov L, Kinori M, Chamlin SL, Wagner A, Kenner-Bell BM, Paller AS, et al. Risk of intraocular and other extracutaneous involvement in patients with cutaneous juvenile xanthogranuloma. Pediatr Dermatol. 2018 May;35(3):329-35.

20 Liang S, Liu YH, Fang K. Juvenile xanthogranuloma with ocular involvement. Pediatr Dermatol. 2009 Mar-Apr; 26(2):232-4.

21 Chantorn R, Wisuthsarewong W, Aanpreung P, Sanpakit K, Manonukul J. Severe congenital systemic juvenile xanthogranuloma in monozygotic twins. Pediatr Dermatol. 2008 Jul;25(4):470-3.

22 Boström J, Janssen G, Messing-Jünger M, JU F, Neuen-Jacob E, Engelbrecht V, et al. Multiple intracranial juvenile xanthogranulomas. Case report. J Neurosurg. 2000 Aug;93(2):335-41.

23 Le Gal FA, Enjolras O, Guillemette J. Tumor on the trunk of a neonate. Pediatr Dermatol. 1996 Feb;13(1):65-8.

24 Yazganoglu KD, Erdem Y, Buyukbabani N, Baykal C. A giant congenital plaque. Pediatr Dermatol. 2012;29(2): 217-8. 
25 Kaur MR, Brundler MA, Stevenson 0, Moss C. Disseminated clustered juvenile xanthogranuloma: an unusual morphological variant of a common condition. Clin Exp Dermatol. 2008 Aug;33(5):575-7.

26 Lamb J, Lain E. Nevoxantho-endothelioma: its relationship to juvenile xanthoma. South Med J. 1937 Jun;30(6): 585-94.

27 National Institutes of Health Consensus Development Conference Statement: neurofibromatosis. Bethesda, Md., USA, July 13-15, 1987. Neurofibromatosis. 1988 May;1(5):172-8.

28 Caputo R. Cutaneous nonhistiocytosis X. In: Freedberg IM, Eisen AZ, Wolff K, editors. Fitzpatrick's dermatology in general medicine. New York: McGraw-Hill, Medical Pub. Division; 1999. p. 1892-902.

29 Ferrari F, Masurel A, Olivier-Faivre L, Vabres P. Juvenile xanthogranuloma and nevus anemicus in the diagnosis of neurofibromatosis type 1. JAMA Dermatol. 2014 Jan;150(1):42-6.

30 Nagayo K, Sakai M, Mizuno N. Juvenile xanthogranuloma with Darier's sign. J Dermatol. 1983 Jun;10(3):283-5.

31 Altman J. Xanthoma disseminatum. Arch Dermatol. 1962 Nov;86(5):582.

32 Altman J, Winkelmann RK. Diffuse normolipemic plane xanthoma. Generalized xanthelasma. Arch Dermatol. 1962 May;85:633-40.

33 Vail JT, Adler KR, Rothenberg J. Cutaneous xanthomas associated with chronic myelomonocytic leukemia. Arch Dermatol. 1985 0ct;121(10):1318. 